\title{
The Retention and Fracture Resistance of Two All-ceramic Post and Core Systems in Anterior Endodontically Treated Teeth
}

\author{
Anahita Safari ${ }^{1}$, Amir Alireza Khaledi ${ }^{1 凶}$, Zeinab Shafiei $^{2}$, Naeeme Rahmani ${ }^{2}$
}

'Department of Prosthodontics, School of Dentistry, Shiraz University of Medical Sciences, Shiraz, Iran. ${ }^{2}$ Student Research Committee, Shiraz University of Medical Sciences, Shiraz, Iran.

\begin{abstract}
Background: In anterior endodontically treated teeth a better esthetic can be achieved by using all-ceramic post -core restorations instead of metal post systems. Maximum retention and fracture resistance of the post are the criteria upon which selection of a post system is based. The aim of this study was to evaluate the fracture resistance and retention of two all-ceramic post and core systems including IPS Empress 2 post-core system and IPS Empress 2 core connected to Zirconium Cosmopost, and comparing these features among the two groups. Material and methods: Eighty extracted maxillary central incisors and canines were divided into two groups of 40 including IPS Empress 2 and zirconium Cosmopost. To evaluate retention, shear force was applied to the cement at the crosshead speed of $0.5 \mathrm{~mm} / \mathrm{min}$; force was applied until the post was detached from the canal. To measure the fracture resistance, the samples were loaded at 45 -degrees to the horizon at the crosshead speed of $5 \mathrm{~mm} / \mathrm{min}$ until fracture occurred.

Result: The mean fracture strength and standard deviation of zirconium Cosmopost and IPS Empress 2 were $302.10 \mathrm{~N}$ (69.922), $151.63 \mathrm{~N}$ (48.661), respectively. The difference between groups was significant statistically $(\mathrm{P}<0.001)$. The zirconium Cosmoposts had lower retention than IPS Empress2 dowel-core, but the difference between groups was insignificant $(p=0.134)$. Conclusion: Within the limitations of the study, it could be concluded that zirconium Cosmopost system may be a candidate for the restoration of anterior endodontically treated teeth, as the high fracture toughness and propitious esthetic feature. [GMJ. 2014;3(2):95-101]
\end{abstract}

Keywords: Ceramic post; Ceramic core; Empress Ceramic; Zirconium-based ceramic; fracture strength; retention

\section{Introduction}

$\mathrm{E}^{\mathrm{s}}$ ndodontically treated teeth are different structurally from un-restored vital teeth and necessitate special care during restoration. The combined loss of structural integrity associated with access preparation, dehydration of dentin after chemo-mechanical preparation, and the excessive pressure during obturation

\section{GMJ}

2013 Galen Medical Journal

Fax: +98 7312227091

PO Box 7461686688

Email:info@gmj.ir compromise these teeth and make them more susceptible to fracture [1,2]. Endodontically treated teeth with insufficient tooth structure are often restored with crowns. If there is inadequate dentin to support a restoration, a post-core is required [3-5].

The primary objective of post and core buildup is to replace the missing coronal tooth structure sufficiently to provide the required

\footnotetext{
Correspondence to:

Amir Alireza Khaledi, Assistant professor, Department of Prosthodontics, School of Dentistry, Shiraz University of Medical Sciences, Shiraz, Iran. Telephone Number: (+98) 9173148061

Email Address :Amiralireza-khaledi@yahoo.com
} 
retention and resistance form for the final restoration [6].

The physical and aesthetic properties of dowel and core restorations are also important factors that can influence the treatment's outcome [7]. Metal posts can create significant esthetic issues as a result of their coloration and interference with natural light transmission through the teeth and the gingival complex; furthermore corrosion reactions can cause metallic taste, oral burning, oral pain, sensitization, and other allergic reactions $[8,9]$. With regard to both esthetic and health concerns, non-metal posts not only render esthetic superiority over metallic posts, but also preclude the possibility of corrosion and reduce the risk of toxicity. Full-ceramic crowns with non-opaque cores (e.g. IPS Empress 2 and Zirconium with medium opacity core material) have the best esthetic appearance. For these advantageous reasons, a wide range of esthetic posts (e.g. ceramic post) have become commercially available [3].

Traditional dental ceramics are brittle, have a low tensile strength, and low fracture toughness while newer all-ceramic materials have improved properties such as high mechanical strength, high toughness, and a Young's modulus similar to that of stainless steel alloy [3, 10]. Several types of these all-ceramic materials have been introduced such as Inceram, zirconium oxide ceramics and pressable ceramics that include IPS Empress 1 and 2.

Maximum retention of the post and the fracture resistance in the restored root are the criteria upon which selection of a post system is based [11]. A study has investigated that fracture resistance of zirconium custom posts was higher than fiber posts and the cast post-cores [12].

Guazzato et al. showed that Inceram posts had higher resistance than IPS Empress Posts [13]. But in another study, Jeong et al. compared 3 different posts and core systems including IPS Empress, Inceram and Cosmopost (zirconium base ceramic). They showed that IPS Empress was the best in fracture resistance [14]. Al-harbi et al. reported Cosmoposts were more retentive than the titanium and composite group [15]. Results from another study showed that Cosmoposts resisted lower than other ceramic posts [16].

Cormier et al. reported that fracture resistance of Cosmoposts was moderate but it was higher than fiber posts [17]. To date, many research articles on ceramic posts have been published $[3,13,14,18]$. However, there is little consensus with regard to their mechanical behavior and reliability, and the factors which would contribute to their optimal application performance. The purpose of this study was to compare the fracture resistance and retention in endodontically treated teeth restored with IPS Empress 2 cores only and two different post systems, including Zirconium Cosmopost and IPS Empress 2.

\section{Materials and Methods}

Eighty maxillary central incisors and canines extracted within the past 2 months were selected from a total of 500 teeth for this experimental study. The teeth had intact crown without caries, restoration, previous root canal therapy (RCT), crack or any attrition and they had comparable length and diameter. Very long $(>32 \mathrm{~mm})$ or very short $(<20 \mathrm{~mm})$ teeth with severe curve were discarded from the study. The fiber-optic trans-illumination was employed to inspect the roots to detect any possible fracture lines. The radiological examination was performed in two buccolingually and mesiodistally dimensions. The teeth with any calcification, internal resorption, open apex, and accessory canal were excluded from the study. Each tooth was labeled with a number from 1 to 80 . The teeth were randomly allocated into two groups, 40 teeth each. For the samples in group A, the dowel-cores were fabricated by IPS Empress 2 and for the teeth in group B, the dowel was made with zirconium and the core was fabricated by IPS Empress 2 material.

Teeth were immersed in 5\% sodium hypochlorite for 15 minutes in order to remove the organic materials from root surfaces. Any remaining tissue was carefully cleaned by using a periodontal curette (Hu-Friedy; USA) and then stored in distilled water.Using diamond discs (Jotta; Germany) mounted on a latch-cut machine under continuous water coolant, the crown was cut by horizontal section perpen- 
dicular to the long axis at the level of $2 \mathrm{~mm}$ incisal to the most coronal point of the cementoenamel junction (CEJ).

\section{Mounting teeth in acrylic block:}

Each tooth was mounted vertically in selfcure acrylic resin in root block former $(2 \times 2 \times 2$ $\mathrm{cm} 3)$ at the level of its CEJ. The periodontal ligament (PDL) should have been simulated in the samples which were used for evaluation of fracture resistance (40). Therefore, teeth were dipped in a molten wax to a depth of $2 \mathrm{~mm}$ below CEJ to provide a 0.2 - to $0.3 \mathrm{~mm}$ - spacer before embedded in the resin. Acrylic resin was poured into the root block former and after the first signs of polymerization, teeth were removed from the resin blocks; wax was eliminated and replaced by silicon impression material injected in the alveolus-like acrylic resin block. The teeth were then reinserted into the acrylic block. During the course of polymerization, the acrylic resin block was cooled in water to avoid dehydration of the dentin and also to prevent the deformation of the resin. The roots were prepared to have 2 $\mathrm{mm}$ ferruled collar, $6^{\circ}$ convergences, and $1 \mathrm{~mm}$ shoulder finish line.

\section{Root canal preparation and obturation:}

After access cavity preparation with a high speed air motor (NSK; Japan), the working length was established $1 \mathrm{~mm}$ shorter than the apex. The canals were instrumented to working length using $\mathrm{K}$-flex file size 40 (Mani; Inc; Japan). A step back flaring technique was performed at $1 \mathrm{~mm}$ increments with Gates Glidden burs (Mani; Inc; Japan) number 2-3. A size $15 \mathrm{~K}$-flex file was passed through the apical foramen of the canal before and after instrumentation to ensure patency. The root canal was irrigated with $15 \mathrm{ml}$ of $1.25 \% \mathrm{Na}-$ $\mathrm{OCl}$ after every file changing. The root canals were dried with sterile paper points before obturation. A size 40 gutta-percha (Gapadentco; LTD; Germany) master cone coated with ZOE sealer was inserted in to the canal. Root canals were obturated using lateral condensation technique with finger spreader (Mani; Inc; Japan). Finally, excess gutta percha were removed and condensed with a hot plugger (Densply; Maillefer; Swiss).
Dowel- space preparation:

A dowel space of $11 \mathrm{~mm}$ length was prepared by using $1.4,1.7 \mathrm{~mm}$ diameter drill corresponding to the Cosmopost (Ivoclar vivadent; Swiss). Before drilling, excess gutta percha was removed by Gates Glidden drills. The canals were cleaned by using air/water spray and then dried by paper points.

\section{Impression technique:}

Indirect impression technique was used for duplicating the dowel space. It was performed in two stages. The primary impression was taken by a partial plastic tray filled with putty (Swiss; Tec; Italy). After removing a thin layer of putty from the impression, additional light silicone (A-silicone Elite; Italy) was injected in to the canal by a special syringe, same material was transported by lentlue (Mani; Inc; Japan) to the canal. Then the impression post, available from the Cosmopost kit, was inserted in to the canal. Finally, impression was taken by the putty tray.

\section{Post and core fabrication method:}

Casts were poured with type IV die stone (GC, Japan). For teeth in group B, Cosmopost with correct size was inserted in the dowel space of die then core modeling was carried out with dental wax. A core pattern was fabricated on a randomly selected tooth using wax, and a condensational silicon mold was fashioned over the core to allow fabrication of standard core patterns on all teeth. After placing sprues, the patterns were burned out. Finally Cosmopost ingot was injected into core mold. In the other group, post and core models were waxed up, burned out and injected with medium opacity IPS Empress Cosmo Ingot (MO, E maks, Swiss). In specimens used for evaluating retention a little hole with $1 \mathrm{~mm}$ diameter was prepared in the core at the stage of wax up that was duplicated in the final core.

\section{Dowel cementation:}

The dentin walls of the dowel space were etched with $37 \%$ phosphoric acid for 40 seconds to eliminate the effect of sealer, rinsed, gently air dried and then ED primer, which is available in the cement kit, was applied in to the canal. After 30 seconds the primer was air 
dried.Finally Panavia cement paste (Kuraray, USA) was mixed according to the manufacturer's instructions. The dowel was coated with cement. Cement was also transported into the canal by lentlue.Posts were positioned in place under firm finger pressure, excess cement was removed, and then oxyguard was applied over the core. For teeth with IPS Empress 2 post, before cementation, dowel surface was etched with $9 \%$ hydrofluoric acid (Ultradent Inc, USA) for 4-5minutes, acid rinsed and air dried, finally silane was applied and air dried after 30 seconds.

\section{Placing specimens on the measuring machine} (Instron Testing Machine):

Before placing the samples on the device, both $\mathrm{A}$ and $\mathrm{B}$ groups were randomly divided into 2 groups, each containing 20 teeth and the fracture resistance would be measured in one category and retention in the other one. In order to measure two variables including fracture resistance and retention, Universal Instron Testing Machine (Zwich, Roell) was used. Before placing the samples, the instrument was calibrated. Position and direction of the samples in the machine was set by the device itself. The specimens were placed in a customized, self-aligning apparatus, which was clamped into place with a vise grip. When assembled, the horizontal rod attached to the upper element of the Instron testing machine was passed through the hole, which was made in the core. To measure the amount of retention, shear force was applied to the cement with speed of $0.5 \mathrm{~mm} / \mathrm{min}$, force was applied until the post was removed from the canal, the force required to remove the post from the canal in its longitudinal axis was reported in Newton to show the amount of retention.

To measure the fracture resistance, the samples were placed in to the device with a 45-degree angle to the horizon and compressive strength was applied to them with the speed of $5 \mathrm{~mm} / \mathrm{min}$. Maximum force resulting in the breakdown of each samples was reported in Newton as the amount of fracture resistance. Student's t-test was used for pairwise comparison. The significance level was set at $p \leq 0.05$. Statistical analysis was performed with SPSS 19.0.

\section{Results}

The mean and standard deviation values for dowel- core retention and fracture resistance of the two various dowel systems are summarized in table 1 .Although the mean retention of zirconium Cosmopost system (125.38 $\mathrm{N})$ was lower than IPS Empress2 restoration $(144.75 \mathrm{~N})$, statistical analysis revealed no significant differences in retention among dowel-core systems $(\mathrm{P}=0.13)$. When groups 1 and 2 were compared the posts in the zirconium Cosmopost group demonstrated higher mean fracture strength and this difference was significant statistically $(\mathrm{P}<0.001)$. None of the specimens broke down from core portion or from core-post interface and there was not any root fracture in samples.

Table 1. Mean and Standard Deviation (Sd) of Fracture Resistance and Retention of Different Post-Core Systems Tested

\begin{tabular}{llllll}
\hline Variable & Dowel Systems & Number Of Specimen & Mean & Sd & P-Value \\
\hline \multirow{2}{*}{ Fracture resistance } & Zirconia & 20 & 302.10 & 69.922 & $<0.001$ \\
& IPS Empress2 & 20 & 151.63 & 48.661 & \\
\hline \multirow{2}{*}{ Retention } & Zirconia & 20 & 125.38 & 36.191 & 0.134 \\
& IPS Empress2 & 20 & 144.75 & 43.544 & \\
\hline
\end{tabular}




\section{Discussion}

Our results indicated that fracture strength of zirconium Cosmopost was higher than IPS Empress2 systems but comparison between the two groups did not reveal any significant difference in retention. The fracture resistance was evaluated using Instron machine. A compressive load at a crosshead speed of $5 \mathrm{~mm} / \mathrm{min}$ was applied at a $135^{\circ}$ angle to the long axis of the tooth .Although, in clinical practice, the velocity of mandibular movement varies considerably, and the impact velocity of the compressive tip was maintained at a crosshead speed of $5 \mathrm{~mm} / \mathrm{min}$-, which is considered as an acceptable average value. Guazzato et al. and Rosentritt et al. showed higher fracture resistance for Cosmopost [9, 13], which is in agreement with the recorded values in this study. This result can be attributed to the brittleness of the ceramic mass and to the fact that Cosmopost has a higher modulus of elasticity (210 GPa).Higher modulus of elasticity resulted in less bending of the dowel/core unit under load [1,9]. Fracture resistance of IPS Empress was higher than zirconium Cosmopost as determined by Jeong et al. [14]; this contradiction may be related to limited number of specimens in their study. Some factors that affect the fracture strength and retention of dowel and core system are the type of cement used, method of cementation, core material and design, crown design and biocompatibility of post material [6]. Cementation with resin cement is reported to increase the retention and fracture resistance of dowels [7]. Mendoza et al. showed that posts cemented with Panavia were significantly more resistant to fracture than those cemented with zinc phosphate. Another investigator found that Panavia provided the highest bond strength for all types of post material. Therefore resin cement (Panavia) was used in the present study. The other factors that affect the fracture resistance of endodontically treated teeth are post diameter, length, design and adaptability, amount of remaining dentin. The amount of tooth structure is a significant factor in determining the fracture resistance of an endodontically treated tooth. It has been shown that endodontically treated teeth restored with posts and cores of different materials and designs tend to exhibit similar fracture resistance when abundant tooth structure remains [6]. The present study simulated a realistic condition of a reduced amount of tooth structure with a $2 \mathrm{~mm}$ height of coronal dentin. With such a design, the compressive load was largely borne by the post and core.

In this study, ferrule collar, a factor that increases resistance to bending force, was created in the restorations and the diameter and length of dowels were standardized $(1.7 \mathrm{~mm}$, $11 \mathrm{~mm}$ ) in all specimens. Although the dowel diameter does not have any influence on dentinal stresses, the increased diameter of the ceramic dowels decreased the bulk of tooth substance and caused root fracture. To prevent root fracture, reduction of the dowel diameter may be considered; however this will reduce the fracture resistance of the ceramic dowel and core under functional loading [7]. It is believed that the use of a rigid material to embed extracted teeth may lead to distorted load values. So, we simulate periodontal ligament by coating the roots with polyvinyl siloxane [1].

Although, IPS Empress is expected to have better retentive features due to etched surface of the post, different fabrication technique in laboratory (waxing up the post) and application of a custom made post rather than a prefabricated one that results in increasing physical adaptation between post surfaces and root walls and increasing mechanical retention, statistical analysis exhibited no significant difference. This finding may be attributed to limitations of the study. The ceramic dowel systems tested were found to have lower retentive values compared with the findings reported by Al-harbi et al. [15]. It is possible that our tested ceramic dowel systems could provide better retention if they had received surface treatments tested in their study (airborne-particle abrasion). According to the Kakehashi's investigation, airborne-particle-abraded Cosmopost provide better retentive values than non-air-particle-abraded dowels [18]. An additional reason for differences in retention might be the type of canal surface treatment used in this research $37 \%$ phosphoric acid in comparing with $17 \%$ EDTA) [1, 18]. However, the present study faced some 
limitations. The results obtained from this study should be interpreted with caution, as teeth were mounted for load testing in a material (acrylic resin) that has limited resiliency. This takes the viable periodontal ligament and resilient alveolar bone, which are crucial parameters on loading, out of equation. Fracture resistance was studied on Instron machine, which did not take into account the oblique, torsional and lateral shearing forces produced during chewing. Another factor is the multidirectional characteristics of masticatory forces, which cannot be duplicated in such machines where a single unidirectional load is applied. It is clear that this type of in vitro loading does not represent the exact situation in vivo [6]. However, the forces were designed on the basis of tests previously reported in the researches involving post and core systems. A single load test was used to investigate the resistance to fracture of endodontically treated teeth. For more meaningful results, future studies should incorporate thermochemical cycling of specimens and fatigue loading; it is important to remember the mean values of forces responsible for failure in the present study and other studies were considerably higher than the maximum physiologic forces acting on teeth. Fatigue stresses may be responsible for fracture with lower forces in the oral cavity [1]. Crown was not prepared for the samples in this study but most of the post and core restorations are clinically followed by full-crown restorations. Cormier et al. stated that a post and core reinforced with full-coverage crown is more fracture resistant than a post alone or a post and core combination [17]. This may be due to the fact that crowns act to distribute applied loads more evenly over the core [6].

In devising the present study, an attempt was made to link an in vitro test to clinical situations. The specimens tested were similar in shape, length and diameter; however the number of specimens was limited. Hence interpreting the differences between fracture resistance of Cosmopost and IPS Empress 2 dowel and cores have been possible to some extent, although extrapolation to the clinical situation must be tempered with caution. Further laboratory research coupled with randomized controlled clinical trials is thus indicated to confirm these results. Also the reader should consider that other factors, such as fatigue, handling and design of the restoration can affect the clinical performance of all-ceramic materials.

\section{Conclusion}

Within the limitations of this study the following conclusions were drawn:

1. The fracture strength of Zirconium Cosmopost system was significantly higher compared with IPS Empress2 system.

2. Zirconium Cosmopost dowel systems had lower retentive values than IPS Empress; but statistical analysis showed no significant difference among the two groups.

3. Pattern of fracture: None of the specimens were breaking down from core portion or from core-post interface.

\section{Acknowledgments}

The authors thank the vice-chancellery of Shiraz University of Medical Sciences, for supporting the research (Grant\#6386). The authers would like to thank Dr. S. Hamedani (DDS, MSc) from the Dental Research Development Center for his editorial assistance, and Dr. M. Vossoughi for the statistical analysis. This article was derived from Z. Shafiee, and N. Rahmani s' thesis.

\section{References}

1. El Guindy J, Fouda MY. Effect of obturating systems, dowel materials, and adhesive luting techniques on the resistance to fracture of endodontically treated teeth. J Prosthodont. 2010;19(7):544-52.
2. Sedgley CM, Messer HH. Are endodontically treated teeth more brittle? J Endod. 1992;18(7):332-5.

3. Ozkurt Z, Iseri U, Kazazoglu E. Zirconia ceramic post systems: a literature review and 
a case report. Dent Mater J. 2010;29(3):23345.

4. Zhi-Yue L, Yu-Xing Z. Effects of post-core design and ferrule on fracture resistance of endodontically treated maxillary central incisors. J Prosthet Dent. 2003;89(4):368-73.

5. Fraga RC, Chaves BT, Mello GS, Siqueira JF, Jr. Fracture resistance of endodontically treated roots after restoration. J Oral Rehabil. 1998;25(11):809-13.

6. Padmanabhan P. A comparative evaluation of the fracture resistance of three different pre-fabricated posts in endodontically treated teeth: An in vitro study. J Conserv Dent. 2010;13(3):124-8.

7. Yalcin E, Cehreli MC, Canay S. Fracture resistances of cast metal and ceramic dowel and core restorations: a pilot study. J Prosthodont. 2005;14(2):84-90.

8. Mekayarajjananonth T, Chitcharus N, Winkler S, Bogert MC. The effect of fiber dowel heights in resin composite cores on restoration failures of endodontically treated teeth. J Oral Implantol. 2009;35(2):63-9.

9. Rosentritt M, Fürer C, Behr M, Lang R, Handel G. Comparison of in vitro fracture strength of metallic and tooth-coloured posts and cores. J Oral Rehabil. 2000;27(7):595601.

10. Al-Wahadni AM, Hussey DL, Grey N, Hatamleh MM. Fracture resistance of aluminium oxide and lithium disilicatebased crowns using different luting cements: an in vitro study. J Contemp Dent Pract. 2009;10(2):51-8.

11. Gluskin AH, Ahmed I, Herrero DB. The aesthetic post and core: unifying radicular form and structure. Pract Proced Aesthet Dent. 2002;14(4):313-21.
12. Abduljabbar T, Sherfudhin H, AlSaleh SA, Al-Helal AA, Al-Orini SS, Al-Aql NA. Fracture resistance of three post and core systems in endodontically treated teeth restored with all-ceramic crowns. KSUJDS. 2012;3(1):33-8.

13. Guazzato M, Albakry M, Ringer SP, Swain MV. Strength, fracture toughness and microstructure of a selection of all-ceramic materials. Part I. Pressable and alumina glass-infiltrated ceramics. Dent Mater. 2004;20(5):441-8.

14. Jeong SM, Ludwig K, Kern M. Investigation of the fracture resistance of three types of zirconia posts in all-ceramic postand-core restorations. Int J Prosthodont. 2002;15(2):154-8.

15. Al-harbi F, Nathanson D. In vitro assessment of retention of four esthetic dowels to resin core foundation and teeth. J Prosthet Dent. 2003;90(6):547-55.

16. Maccari PC, Conceicao EN, Nunes MF. Fracture resistance of endodontically treated teeth restored with three different prefabricated esthetic posts. J Esthet Restor Dent. 2003;15(1):25-30.

17. Cormier CJ, Burns DR, Moon P. In vitro comparison of the fracture resistance and failure mode of fiber, ceramic, and conventional post systems at various stages of restoration. J Prosthodont. 2001;10(1):2636.

18. Kakehashi Y, Lüthy H, Naef R, Wohlwend A, Schärer P. A new all-ceramic post and core system: clinical, technical, and in vitro results. Int J Periodontics Restorative Dent. 1998;18(6):586-93. 